\title{
Expression levels of $F G F R 3$ as a prognostic marker for the progression of primary pT1 bladder cancer and its association with mutation status
}

\author{
HO WON KANG ${ }^{1}$, YE-HWAN KIM ${ }^{1}$, PILDU JEONG ${ }^{1}$, CHEOL PARK ${ }^{1}$, WON TAE KIM ${ }^{1}$, DONG HEE RYU², \\ EUN-JONG CHA ${ }^{3}$, YUN-SOK HA ${ }^{4}$, TAE-HWAN KIM ${ }^{4}$, TAE GYUN KWON ${ }^{4}$, SUNG-KWON MOON ${ }^{5}$, \\ YUNG HYUN CHOI ${ }^{6}$, SEOK-JOONG YUN ${ }^{1}$ and WUN-JAE KIM ${ }^{1}$ \\ Departments of ${ }^{1}$ Urology, ${ }^{2}$ Surgery and ${ }^{3}$ Biomedical Engineering, Chungbuk National University Hospital, \\ College of Medicine, Chungbuk National University, Cheongju, Chungcheongbuk-do 28644; ${ }^{4}$ Department of Urology, \\ School of Medicine, Kyungpook National University Medical Center, Daegu 41404; ${ }^{5}$ Department of Food \\ and Biotechnology, Chungang University, Seoul 06974; ${ }^{6}$ Department of Biomaterial Control, \\ Dong-Eui University, Busan 47340, Republic of Korea
}

Received March 29, 2016; Accepted April 28, 2017

DOI: $10.3892 / \mathrm{ol} .2017 .6621$

\begin{abstract}
The present study examined the utility of fibroblast growth factor receptor 3 (FGFR3) mutation status and gene expression as a prognostic marker in primary pT1 bladder cancer (BC). A total of 120 patients with primary pT1 BC were enrolled. FGFR3 mutation status was determined by direct sequencing and FGFR3 mRNA expression level was determined by reverse transcription-quantitative polymerase chain reaction (RT-qPCR) analysis. The results were compared with the clinicopathological parameters, and the prognostic value of FGFR3 was evaluated by Kaplan-Meier analysis and a multivariate Cox regression test. FGFR3 mutations were identified in 48/120 (40.0\%) patients with pT1 BC. FGFR3 mRNA expression level was significantly higher in those with BC harboring FGFR3 mutations $(\mathrm{P}<0.001)$. Low FGFR3 expression level was associated with high-grade tumors and cancer progression $(\mathrm{P}=0.006$ and $\mathrm{P}=0.001)$, whereas $F G F R 3$ mutation status was not associated with cancer progression. Kaplan-Meier analysis revealed a similar result (log-rank, $\mathrm{P}<0.001)$. Multivariate analysis identified low FGFR3 expression level (odds ratio, 3.300; 95\% confidence interval, 1.310-8.313; $\mathrm{P}=0.011)$ as an independent predictor of cancer progression. Stratification by exon site of FGFR3 mutations
\end{abstract}

Correspondence to: Professor Seok-Joong Yun or Professor Wun-Jae Kim, Department of Urology, Chungbuk National University Hospital, College of Medicine, Chungbuk National University, 776 1sunhwan-ro, Seowon-gu, Cheonju, Chungcheongbuk-do 28644, Republic of Korea

E-mail: sjyun@chungbuk.ac.kr

E-mail:wjkim@chungbuk.ac.kr

Key words: bladder cancer, fibroblast growth factor receptor 3, mutation, expression, prognosis yielded significant differences in mRNA expression level. None of the patients with BC harboring FGFR3 mutations in exon 9 demonstrated disease progression. The mRNA expression level of the FGFR3 gene may be used to precisely identify subsets of patients with pT1 BC that have a relatively better prognosis. The prognostic influences of FGFR3 mutations may be modulated by the exon site of FGFR3 mutations.

\section{Introduction}

Urothelial cell carcinoma (UCC) of the bladder is the fifth most common type of tumor and the second most common cause of mortality in patients with genitourinary tract malignancies in developed countries (1). Bladder cancer (BC) comprises two long-recognized disease entities, non-muscle invasive bladder cancer (NMIBC) and muscle invasive bladder cancer (MIBC), which have distinct molecular features and clinical outcomes (2). Although 70-80\% of patients are diagnosed with NMIBC at the time of initial presentation, high recurrence rates (50-70\%) have been observed in these patients (3). Furthermore, about one-third of recurrent cases will progress to MIBC and eventually succumb to the disease (4). The present study acknowledges that the biology of tumors, particularly pT1 bladder tumors, of a similar stage and grade can vary greatly. Thus, identifying the patients that are at risk of developing MIBC and the patients that are not is important for appropriate disease management.

Currently, pathological analyses (including clinical stage and tumor grade) are key determinants for risk assessment and therapeutic decision making in BC (5). However, none of the predictive values derived from conventional histopathological parameters have demonstrated sufficient sensitivity or specificity for detecting, monitoring and determining the prognosis of $\mathrm{BC}(5,6)$. These limitations have led to numerous previous studies that aimed to identify molecular markers that enable clinicians to classify BCs in more detail, thereby enabling appropriate selection of the optimal treatment 
regimen (2). Recent genome-wide expression and sequencing studies identified the genes and signaling pathways that are key drivers of urothelial cancer and revealed a more complex picture comprising multiple molecular subclasses that traverse conventional grade and stage groupings $(7,8)$. Numerous studies have revealed that low-grade noninvasive and high-grade invasive BC are genetically and clinically disparate entities $(9,10)$. Low-grade noninvasive bladder tumors are characterized by gain-of-function mutations, which mainly affect classical oncogenes including fibroblast growth factor receptor 3 (FGFR3) and Harvey rat sarcoma viral oncogene homolog genes, whereas invasive tumors are characterized by loss-of-function mutations resulting in inactivation of tumor suppressors including $p 53, R B$, and phosphate and tensin homolog $(11,12)$. FGFR3 belongs to a family of structurally associated tyrosine kinase receptors that are involved in numerous aspects of embryogenesis and tissue homeostasis, as well as being implicated in the tumorigenesis of bladder and other urothelial types of cancer, multiple myeloma and cervical cancer (13-15). Mutated FGFR3 is constitutively activated and induces a number of oncogenic signaling pathways, including the RAS/mitogen activated protein kinases (MAPK), phospholipase Cc1 (PLCc1), phosphoinositide 3-kinase (PI3K) and signal transducer and activator of transcription (STAT) signaling pathways (11,16-18). Activating mutations in FGFR3 genes are associated with genetically stable Ta and low-grade $\mathrm{BC}$, which represent the favorable BC pathway (19). Activating mutations of FGFR 3 are observed in $\leq 70 \%$ of NMIBC cases, whereas overexpression of a wild-type receptor has been revealed in $\sim 40 \%$ of patients with invasive disease (20). Although numerous studies identified associations between FGFR3 mutation status and pathological phenotype, the prognostic implications of these activating mutations has not been clearly established (20-23). To the best of our knowledge, no previous studies have undertaken a comprehensive analysis of FGFR3 mutation status and gene expression as prognostic markers in primary $\mathrm{pT} 1 \mathrm{BC}$.

In the present study, the association between FGFR3 gene expression level, mutation status and pathological phenotype in primary pT1 BC tissues was examined. Of note, the present study also evaluated the implications of $F G F R 3$ as a prognostic indicator for $\mathrm{pT} 1 \mathrm{BC}$.

\section{Materials and methods}

Study population and follow-up protocols. Tissue samples were obtained from 151 consecutive patients with primary pT1 BC who underwent transurethral resection (TUR) for histologically diagnosed transitional cell carcinomas between January 1996 and December 2008 at Chungbuk National University Hospital (South Korea). The tissue samples for the present study were provided by Chungbuk National University Hospital, a member of the National Biobank of Korea, which is supported by the Ministry of Health, Welfare and Family Affairs. All tumors were macrodissected within 15 min of surgical resection, fresh-frozen in liquid nitrogen and stored at $-80^{\circ} \mathrm{C}$ until use. Each patient was independently reviewed by a genitourinary pathologist who was unaware of how the clinical data were to be used. All patients received six cycles of induction Bacillus Calmette-Guerin (BCG) therapy (12.5 mg of Tice strain BCG in $50 \mathrm{ml}$ of physiological bacteriostatic-free saline solution), according to European Association of Urology guidelines, and were confirmed to be disease free 3 months following transurethral resection of the bladder tumor (TURB) following BCG induction therapy. In order to reduce confounding factors affecting the analyses and to delineate a more homogenous study population, patients undergoing immediate postoperative therapy with single-dose mitomycin $\mathrm{C}(\mathrm{n}=8)$ or BCG maintenance therapy $(\mathrm{n}=12)$, or those diagnosed with a concomitant carcinoma in situ $(\mathrm{n}=6)$, were excluded from the study. To avoid the risk of under staging, cases where bladder muscle was not clearly identifiable $(n=5)$ were also excluded. Therefore, 120 primary pT1 BC cases were finally used for analysis. The study cohort included 97 males and 23 females. The mean age of patients was 65.93 years (range, 24-88 years).

Tumors were staged according to the 2002 tumornode-metastasis classification system and the 1973 World Health Organization grading system $(5,24)$. When a BC specimen did not include sufficient muscle or when a grade 3 tumor was detected, a second-look TURB was systematically conducted 2-4 weeks after the initial resection. Following initial TURB, each patient was monitored according to standard guidelines (5). Standard follow-up included cystoscopy and urinary cytology at 3-monthly intervals for 2 years, then 6-monthly intervals for 2 years and yearly intervals thereafter. Radiographic evaluation including chest and abdominal computed tomography was performed on an annual basis for evaluation of the upper urinary tract and early detection of metastasis. Recurrence was defined as the recurrence of primary NMIBC at a lower or equivalent pathological stage, and progression was defined as muscular invasion, increased tumor grade or metastatic disease.

Good clinical practice protocols. The present study was performed in agreement with the applicable laws and regulations, good clinical practice and the ethical principles described in the Declaration of Helsinki. The study protocol was approved by the Ethics Committee of Chungbuk National University (IRB approval no. 2010-01-001; Cheongju, Korea). Written informed consent was obtained from all patients prior to enrollment in the present study. Sample collection and analysis procedures were also approved by the Institutional Review Board of Chungbuk National University.

Analysis of FGFR3 mutations. Genomic DNA was isolated from frozen tumor tissue specimens using the Wizard Genomic DNA Purification System kit (Promega Corporation, Madison, WI, USA), according to the manufacturers protocol. The FGFR3 gene sequence was obtained from the NCBI database (http://www.ncbi.nlm.nih.gov/gene/2261). Three regions (exons 7,9 and 14) harboring 11 frequent oncogenic FGFR3 mutations were simultaneously amplified by polymerase chain reaction (PCR). Detailed PCR methods were performed as previously described (25). The PCR products were purified and sequenced using the BigDye Terminator v3.1 Cycle Sequencing kit and an ABI 3730xl automatic sequencer (both from Applied Biosystems; Thermo Fisher Scientific, Inc., Waltham, MA, USA). 
Table I. Baseline characteristics of the patients.

\begin{tabular}{lc}
\hline Parameters & Number $(\%)$ \\
\hline Mean age \pm SD, years (range) & $65.93 \pm 12.93(24-88)$ \\
Median follow-up, months (IQR) & $69.3(29.2-103.1)$ \\
Gender & \\
Male & $97(80.8)$ \\
Female & $23(19.2)$ \\
Smoking (ex-or current) & $51(42.5)$ \\
Tumor size (cm) & \\
$\leq 3$ & $49(40.8)$ \\
$\geq 3$ & $71(59.2)$ \\
Multiplicity & \\
Single & $54(45.0)$ \\
Multiple & $66(55.0)$ \\
Grade & \\
I & $17(14.2)$ \\
II & $79(65.8)$ \\
III & $24(20.0)$ \\
Recurrence & $61(50.8)$ \\
Progression & $20(16.7)$ \\
\hline
\end{tabular}

$\mathrm{SD}$, standard deviation; IQR, interquartile range.

Table II. Association between FGFR3 mutation status and mRNA expression level in pT1 BC.

\begin{tabular}{lccc}
\hline $\begin{array}{l}\text { FGFR3 } \\
\text { mutation }\end{array}$ & $\begin{array}{c}\text { Number } \\
(\%)\end{array}$ & $\begin{array}{c}\text { mRNA expression } \\
\text { level, median } \\
\left(\text { IQR; } \times 10^{4} \text { copies } / \mu \mathrm{g}\right)\end{array}$ & P-value \\
\hline Wild-type & $72(60.0)$ & 154.23 & $<0.001^{\mathrm{a}}$ \\
Mutant & $48(40.0)$ & $(61.16-419.49)$ & \\
& & 728.38 & \\
R248C & 11 & & \\
S249C & 13 & \\
G370C & 2 & \\
S371C & 2 & \\
Y373C & 16 & \\
A391E & 1 & \\
K650M & 1 & \\
K650E & 2 & \\
K650T & 1 & \\
\hline
\end{tabular}

aP-value was based on the Mann-Whitney U test for wild-type vs. mutant. One patient exhibited a concurrent mutation in R248C and K650E. FGFR3, fibroblast growth factor receptor 3; BC, bladder cancer.

Analysis of FGFR3 mRNA expression level. Total RNA was extracted from tissue samples using TRIzol ${ }^{\circledR}$ reagent (Invitrogen, Thermo Fisher Scientific, Inc.), according to the manufacturer's protocol. cDNA was prepared from $1 \mu \mathrm{g}$ RNA using random primers and a First-Strand cDNA Synthesis kit (GE Healthcare Life Sciences, Chalfont, UK). To quantify the expression levels of FGFR3, RT-qPCR amplification was performed using a Rotor Gene 6000 instrument (Corbett Life Science; Qiagen, Inc., Valencia, CA, USA). RT-qPCR assays were performed in micro-reaction tubes (Corbett Life Science; Qiagen, Inc.) containing SYBR Premix EX Taq (Takara Biotechnology Co., Ltd., Dalian, China). The following primers were used to amplify $F G F R 3$ (146 base pairs): Sense, 5'-CGTACTGTGCCACTTCAGTG-3' and antisense, 5'-CCAGCAGCTTCTTGTCCATC-3'. The PCR reaction was performed in a final volume of $10 \mu \mathrm{l}$, comprising $5 \mu \mathrm{l}$ of $2 \mathrm{X}$ SYBR Premix EX Taq buffer, $0.5 \mu 1$ of each 5' and 3'primer $(10 \mathrm{pM} / \mu \mathrm{l})$ and $1 \mu \mathrm{l}$ sample cDNA. The products were purified using a QIAquick Extraction kit (Corbett Life Science; Qiagen), quantified in a spectrometer (MBA 2000; Perkin Elmer, Inc., Waltham, MA, USA) and sequenced using an automated laser fluorescence sequencer (ABI PRISM 3100 Genetic Analyzer; Applied Biosystems, Foster City, CA, USA). A known concentration of the PCR product was then 10 -fold serially diluted from 100 to $0.1 \mathrm{pg} / \mu \mathrm{l}$ and used to establish a standard curve. The RT-qPCR conditions were 1 cycle at $96^{\circ} \mathrm{C}$ for $20 \mathrm{sec}$, followed by 40 cycles of $2 \mathrm{sec}$ at $96^{\circ} \mathrm{C}$ for denaturation, $15 \mathrm{sec}$ at $60^{\circ} \mathrm{C}$ for annealing and $15 \mathrm{sec}$ at $72^{\circ} \mathrm{C}$ for extension. The melting program was performed at $72-95^{\circ} \mathrm{C}$ with a heating rate of $1^{\circ} \mathrm{C}$ per $45 \mathrm{sec}$. Spectral data were captured and analyzed using Rotor-Gene Real-Time Analysis Software 6.0 Build 14 (Qiagen, Inc.). All samples were run in triplicate. GAPDH was used as an endogenous RNA reference gene. Relative quantification of gene expression was performed using the $2^{-\Delta \Delta C q}$ calculation formula, based on $\mathrm{Cq}$ values for target and reference genes (26). The gene expression was normalized to the expression of GAPDH.

Statistical analysis. Continuous variables are expressed as the median and interquartile range (IQR). Differences between variables demonstrating a continuous distribution across dichotomous categories were assessed using the Mann-Whitney $U$ test. The Fisher's exact and $\chi^{2}$ tests were used to evaluate associations between categorical variables. The Kaplan-Meier method was used to estimate time to recurrence and progression, and differences were assessed using the log-rank test. The prognostic value of FGFR mutation status and gene expression level was analyzed using univariate and multivariate Cox's regression test. FGFR3 mRNA expression level was classified according to the quartiles of the range, and the lowest quartile $\left(<107.70 \times 10^{4}\right.$ copies $\left./ \mu \mathrm{g}\right)$ was assigned to the reference group for regression analysis. $\mathrm{P}<0.05$ was considered to indicate a statistically significant difference. All reported P-values are two-sided. All statistical analyses were performed using SPSS version 20.0 software (IBM SPSS, Armonk, NY, USA).

\section{Results}

Baseline characteristics. The baseline characteristics of the 120 patients with primary pT1 BC are presented in Table I. The study cohort included 97 males and 23 females. The mean age of patients was 65.93 years (range, $24-88$ years). The histological 
Table III. Association between FGFR3 mutation status, mRNA expression level and clinicopathological features in pT1 BC.

FGFR3 mutation

\begin{tabular}{|c|c|c|c|c|c|}
\hline \multirow[b]{2}{*}{ Parameters } & & \multirow[b]{2}{*}{ P-value } & \multirow{2}{*}{$\begin{array}{c}\text { mRNA expression level, } \\
\left.\text { median (IQR; } x 10^{4} \text { copies } / \mu \mathrm{g}\right)\end{array}$} & \multirow[b]{2}{*}{ P-value } \\
\hline & Wild-type $(n=72)$ & Mutation $(n=48)$ & & & \\
\hline Gender & & & $0.350^{\mathrm{a}}$ & & $0.772^{\mathrm{b}}$ \\
\hline Male & $56(77.8)$ & $41(85.4)$ & & $304.04(100.58-848.86)$ & \\
\hline Female & $16(22.2)$ & $7(14.6)$ & & $263.43(128.46-514.87)$ & \\
\hline Tumor size & & & $0.349^{\mathrm{a}}$ & & $0.056^{\mathrm{b}}$ \\
\hline$<3 \mathrm{~cm}$ & $32(44.4)$ & $17(35.4)$ & & $219.23(69.98-545.59)$ & \\
\hline$\geq 3 \mathrm{~cm}$ & $40(55.6)$ & $31(64.6)$ & & 369.27 (146.93-956.35) & \\
\hline Multiplicity & & & $0.708^{\mathrm{a}}$ & & $0.945^{\mathrm{b}}$ \\
\hline Single & $31(43.1)$ & $23(47.9)$ & & $272.10(107.73-1116.69)$ & \\
\hline Multiple & $41(56.9)$ & $25(52.1)$ & & 336.62 (98.00-727.14) & \\
\hline Grade & & & $0.001^{\mathrm{a}}$ & & $0.006^{\mathrm{c}}$ \\
\hline I & $6(8.3)$ & $11(22.9)$ & & $453.92(242.84-1076.38)$ & \\
\hline II & $44(61.1)$ & $35(72.9)$ & & 342.25 (119.95-1038.87) & \\
\hline III & $22(30.6)$ & $2(4.2)$ & & $130.04(33.47-306.05)$ & \\
\hline Recurrence & & & $0.264^{\mathrm{a}}$ & & $0.856^{\mathrm{b}}$ \\
\hline No & $32(44.4)$ & $27(56.2)$ & & $304.04(127.82-685.67)$ & \\
\hline Yes & $40(55.6)$ & $21(43.8)$ & & $286.62(78.73-869.24)$ & \\
\hline Progression & & & $0.050^{\mathrm{a}}$ & & $0.001^{\mathrm{b}}$ \\
\hline No & $56(77.8)$ & $44(91.7)$ & & $367.78(132.51-883.11)$ & \\
\hline Yes & $16(22.2)$ & $4(8.3)$ & & 78.73 (22.57-302.03) & \\
\hline
\end{tabular}

P-values were obtained from ${ }^{\mathrm{a}}$ Fisher's exact test, ${ }^{\mathrm{b}} \mathrm{Mann}$-Whitney U-test or ${ }^{\mathrm{C}}$ Kurskal-Wallis Test. FGFR3, fibroblast growth factor receptor 3; $\mathrm{IQR}$, interquartile range; $\mathrm{SD}$, standard deviation; $\mathrm{BC}$, bladder cancer.

grade distribution was as follows: $14.2 \%$ grade I; $65.8 \%$ grade II and $20.0 \%$ grade III. A total of 61 patients (50.8\%) exhibited recurrent disease and progression was observed in 20 patients (16.7\%) during a median follow-up period of 69.3 months (IQR, 29.2-103.1 months). The median intervals for recurrence and progression were 20.7 months (range, 6.4-133.6) and 43.0 months (range, 6.6-115.4), respectively.

Of the 20 progressive cancers, 4 cases demonstrated an increased tumor grade within the equivalent pathological stage and 16 cases progressed to MIBC. A total of 15 cases underwent radical cystectomy and the other cases received palliative chemotherapy or radiation therapy: Of those, 8 patients succumbed to $\mathrm{BC}$.

Association between FGFR3 mutation status and mRNA expression level in pT1 BC tissues. FGFR3 mutations were identified in 48/120 (40.0\%) patients with pT1 BC. The most common mutations were Y373C, R249C and R248C, which were observed in 16, 13 and 11 cases, respectively. FGFR3 mRNA expression level was significantly higher in FGFR3 mutant BC compared with in FGFR3 wild-type BC $(\mathrm{P}<0.001)$. The median FGFR3 mRNA expression levels for mutant and wild-type BC were $728.38 \times 10^{4}$ (IQR, 282.23-1287.61) copies/ $\mu \mathrm{g}$ and $154.23 \times 10^{4}$ (IQR, 61.16-419.49) copies/ $\mu$ g, respectively (Table II).

Association between FGFR3 mutation status, mRNA expression level and clinicopathological features in pT1 BC tissues.
BC harboring wild-type FGFR3 and low FGFR3 expression level was associated with high-grade tumors $(\mathrm{P}=0.006)$. However, there were no significant differences in FGFR3 mutation status or mRNA expression level according to other clinicopathological parameters, including age, tumor size and multiplicity (all P>0.05; Table III).

Prognostic value of FGFR3 mutation status and mRNA expression level in pT1 BC tissues. There were no significant differences in FGFR3 mutation status or mRNA expression level in terms of tumor recurrence $(\mathrm{P}=0.264$ and $\mathrm{P}=0.856$, respectively). Patients who experienced cancer progression exhibited significantly lower expression levels of FGFR3 mRNA compared with patients who did not $(\mathrm{P}=0.001$; Table III). Kaplan-Meier analysis revealed that patients with high FGFR3 mRNA expression level demonstrated better progression-free survival compared with those with lower expression levels of FGFR3 mRNA (log-rank, P<0.001; Fig. 1).

Multivariate Cox regression analysis identified low FGFR3 expression level (odds ratio, 3.300; 95\% confidence interval, 1.310-8.313; $\mathrm{P}=0.011$ ) and tumor grade III (odds ratio, 2.623; $95 \%$ confidence interval, $1.161-5.927 ; \mathrm{P}=0.020)$ as an independent predictor of cancer progression (Table IV).

Association between FGFR3 mutation site, mRNA expression level and cancer progression in pT1 BC. When FGFR3 mutations were categorized by exon site, mutations in exons 7 and 9 


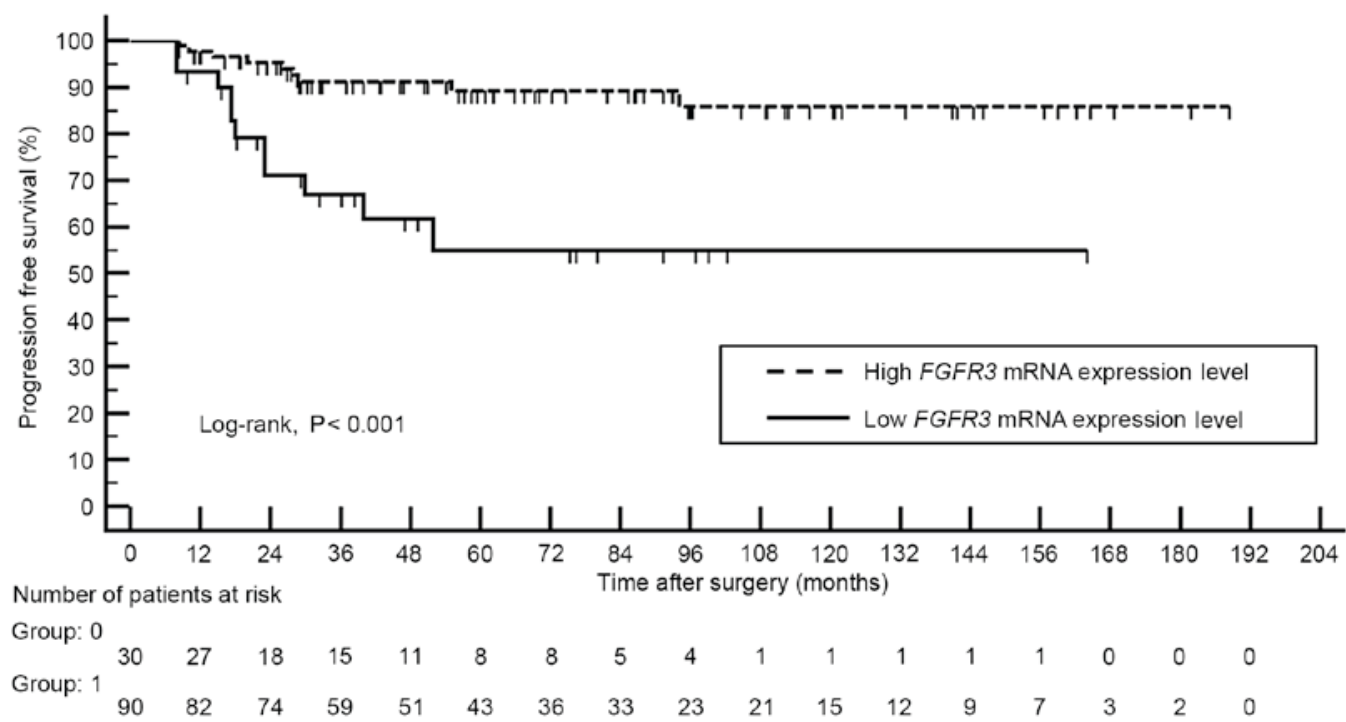

Figure 1. Kaplan-Meier curves for predicting progression-free survival according to $F G F R 3 \mathrm{mRNA}$ expression level. Cutoff value was $107.70 \mathrm{x} 10^{4} \mathrm{copies} / \mu \mathrm{g}$. $F G F R 3$, fibroblast growth factor receptor 3 .

demonstrated significantly high mRNA expression levels compare with the wild type $\mathrm{BC}$ (each $\mathrm{P}<0.001$; Fig. 2). By contrast, mutations located in exon 14 did not reveal a significant difference in FGFR 3 mRNA expression level compared with in the wild type BC. None of the patients with BC harboring FGFR 3 mutation in exon 9 demonstrated disease progression or metastasis (Fig. 3).

\section{Discussion}

The present study examined the utility of $F G F R 3$ mutations and $F G F R 3$ gene expression as prognostic markers in primary pT1 BC. FGFR 3 mRNA expression was associated with the presence of $F G F R 3$ mutation. FGFR 3 mRNA expression level was an independent predictor of progression. FGFR 3 mutation was significantly associated with tumor grade but not with cancer progression.

FGFR3 is a receptor tyrosine kinase implicated in the tumorigenesis of numerous types of myeloma, cervical cancer and urothelial carcinoma (13). There are two mechanisms that cause abnormal activation of FGFR3: Translocation of chromosome 4 to chromosome 14 (leading to overexpression) and activation of point mutations in the FGFR3 gene (11). Activating mutations of FGFR3 are observed in the majority of NMIBCs (35.5-78.1\%), and the overexpression of a wild-type receptor has been identified in $~ 40 \%$ of MIBC (21). Constitutive (ligand-independent) receptor activation occurs most commonly by substitution of a wild-type residue within the extracellular domain of FGFR3 with a cysteine residue, resulting in dimerization and subsequent stimulation of tyrosine kinase activity $(11,27)$. This in turn induces a number of different oncogenic signaling pathways, including the RAS/MAPK, PLCc1, PI3K and STAT pathways $(7,17,18)$. FGFR3 point mutations are found almost exclusively in exons 7, 10 and 15 (19). The most frequent extracellular domain-activating mutations are R248C and S249C, and transmembrane domain mutations include $\mathrm{G} 372 \mathrm{C}$ and $\mathrm{Y} 375 \mathrm{C}$; other mutations occur at low frequencies $(6,16)$. The frequency of FGFR 3 mutations at these hot spots in the present study's cohort were similar to those described in previous studies (23). Cappellen et al (14) conducted the first study examining FGFR3 involvement in bladder tumors. Since then, numerous studies have been performed to better understand the potential role of mutant $F G F R 3$ as an oncogenic driver, particularly in BC $(18-21,23,28)$. Previous studies also demonstrated that FGFR 3 mutations are associated with genetically stable Ta and low-grade BC, which represents the favorable BC pathway (20). Activating mutations in the FGFR 3 gene have been reported in $\leq 75 \%$ of low-grade and low-stage BC, but are absent or rare in carcinoma in situ and MIBC (29). The results presented in the present study confirm previous studies demonstrating that the presence of $F G F R 3$ mutations is significantly associated with low tumor grade (23). The association between FGFR3 mutations and pathological phenotype has been well established, but the prognostic significance of $F G F R 3$ mutations in $\mathrm{BC}$ remains poorly defined (20). A previous study by van Rhijn et al (19) reported that $F G F R 3$ mutations were an independent predictor of recurrence in NMIBC. BC recurrence was more common in patients whose initial tumor was classified as wild-type rather than as harboring a mutant $F G F R 3$ gene. Conversely, a large prospective study of 772 patients revealed a significantly higher rate of recurrence in patients harboring an FGFR3 mutation compared with in those with a FGFR 3 wild-type tumor (22). Following stratification according to tumor stage and grade, the prognostic value of the FGFR 3 mutation in terms of tumor recurrence appeared to be restricted to pTaG1 tumors, and a previous study suggested that additional molecular alterations within higher grade/stage tumors overrode the association between FGFR 3 mutation and prognosis (22). In addition, there is certain evidence supporting the prognostic value of FGFR 3 mutations for predicting the risk of progression $(23,30)$. The exact prognostic role of these mutations with respect to NMIBC progression has not yet been fully elucidated; however, two recently published studies suggested the possibility of a progression-associated prognostic indicator for NMIBC $(4,23,30)$. A study by van Rhijn et al $(23)$ 


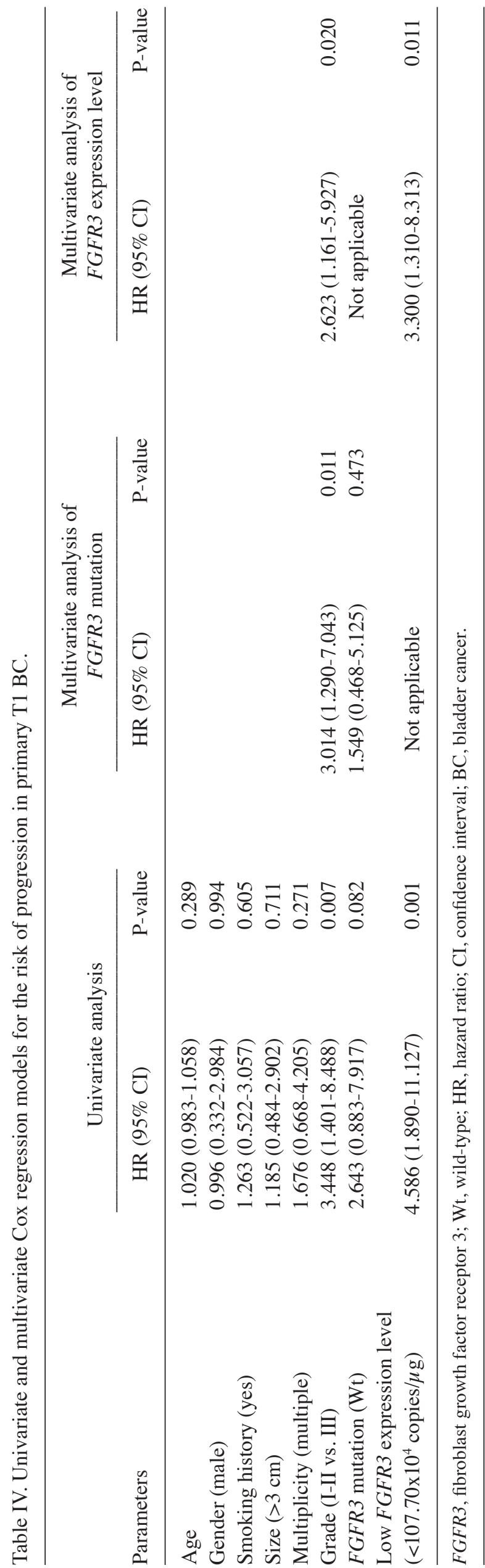

examined the distribution and clinical outcome of FGFR3 and P53 alterations in 132 patients with primary pT1 BC. Multivariate analyses revealed that FGFR3 mutation status was a significant prognostic factor for progression. Another study by Burger et al (30) revealed that FGFR3 status did discriminate progressors from non-progressors within a subset of patients with high-grade BC. Although the design and outcome evaluations of the present study were similar to previous studies, the present study demonstrated a different result in which FGFR3 mutation status did not have prognostic significance in terms of tumor recurrence or progression. Numerous factors may account for these discrepant results. Firstly, the resent study adopted strict exclusion criteria to eliminate possible interference. To delineate a more homogenous study population, patients who received intravesical chemotherapy or BCG maintenance therapy or those diagnosed with a concomitant carcinoma in situ were excluded from the study. Although van Rhijn et al (23) specifically analyzed patients with primary $\mathrm{pT} 1 \mathrm{BC}$ who received $\mathrm{BCG}, 35 \%$ of $\mathrm{BC}$ cases were concomitant carcinoma in situ, which frequently resembles a muscle invasive disease due to its aggressive biological features. It is also possible that the participants in the present study had different tumor characteristics. In the study by Burger et al (30), the majority of patients exhibited a relatively favorable tumor characteristic, $81 \%$ of pTa tumor and $89 \%$ of G1-2 tumor, whereas van Rhijn et al (23) enrolled patients with a primary diagnosis of pT1 and majority of the patients exhibited high-grade tumors $(80 \%)$. The results of the present study were also acquired from a homogenous population with a primary diagnosis of pT1 and $65 \%$ of T1 BC was Grade II. Tumor staging and grading were reassigned by one genitourinary pathologist; however, only $20 \%$ of T1 BC was assigned to grade III. The progression rate of the $\mathrm{BC}$ cohort was lower compared with in the study by van Rhijn et al (23) and this may be due to these tumor characteristics. In the present study, FGFR3 mutant BC was associated with a favorable tumor grade and high FGFR3 mRNA expression level, but it did not affect prognostic impact on progression. Further large cohort collaboration studies should be performed to confirm the prognostic role of FGFR3 mutation in pT1 BC.

The majority of previous studies focused on FGFR 3 mutation status and protein expression level with respect to pathological phenotype and oncological outcome $(16,19,21,23,28)$. At present, little is known about the association between mutation status and FGFR3 mRNA expression level in BC (28). A study by Bernard-Pierrot et al (27) investigated the association between FGFR3 mRNA expression levels and FGFR3 status, and demonstrated that high expression levels of FGFR3 correlated with the presence of a mutated FGFR 3 gene. However, the level of FGFR3 mRNA was determined by semi-quantitative radioactive $\mathrm{RT}$-qPCR, and they did not identify a significant association between FGFR3 mRNA expression levels and tumor characteristics. Furthermore, as far as can be ascertained, no previous study has addressed the prognostic implications of FGFR3 mRNA expression level in BC. The present study revealed that lower FGFR3 mRNA expression level was an independent predictor of progression. FGFR3 mRNA expression level may be useful for predicting the outcome of high-risk refractory tumors in $\mathrm{pT} 1 \mathrm{BC}$ prior to their progression. The present study further analyzed the FGFR3 mRNA 


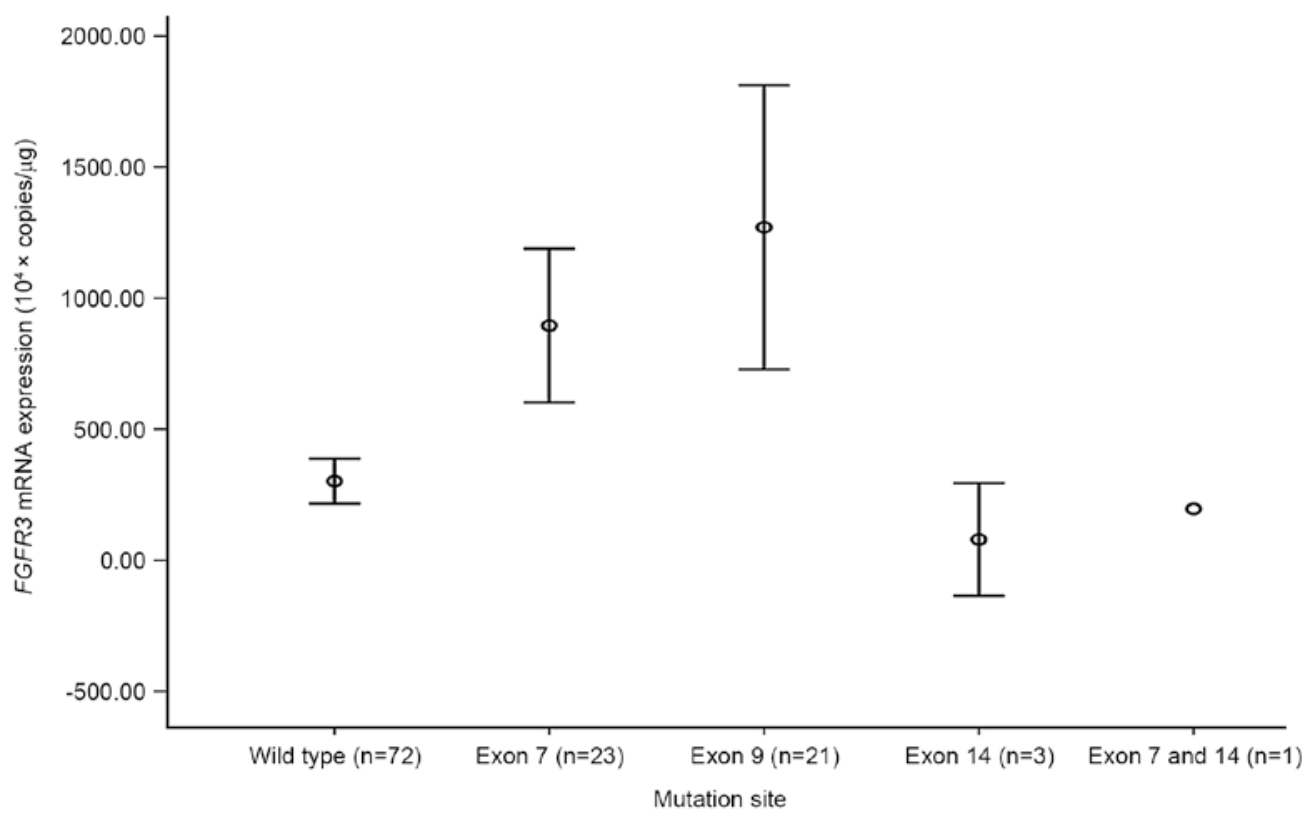

Figure 2. Differences in FGFR3 mRNA expression level according to FGFR3 mutation status and mutation exon site. FGFR3, fibroblast growth factor receptor 3 .

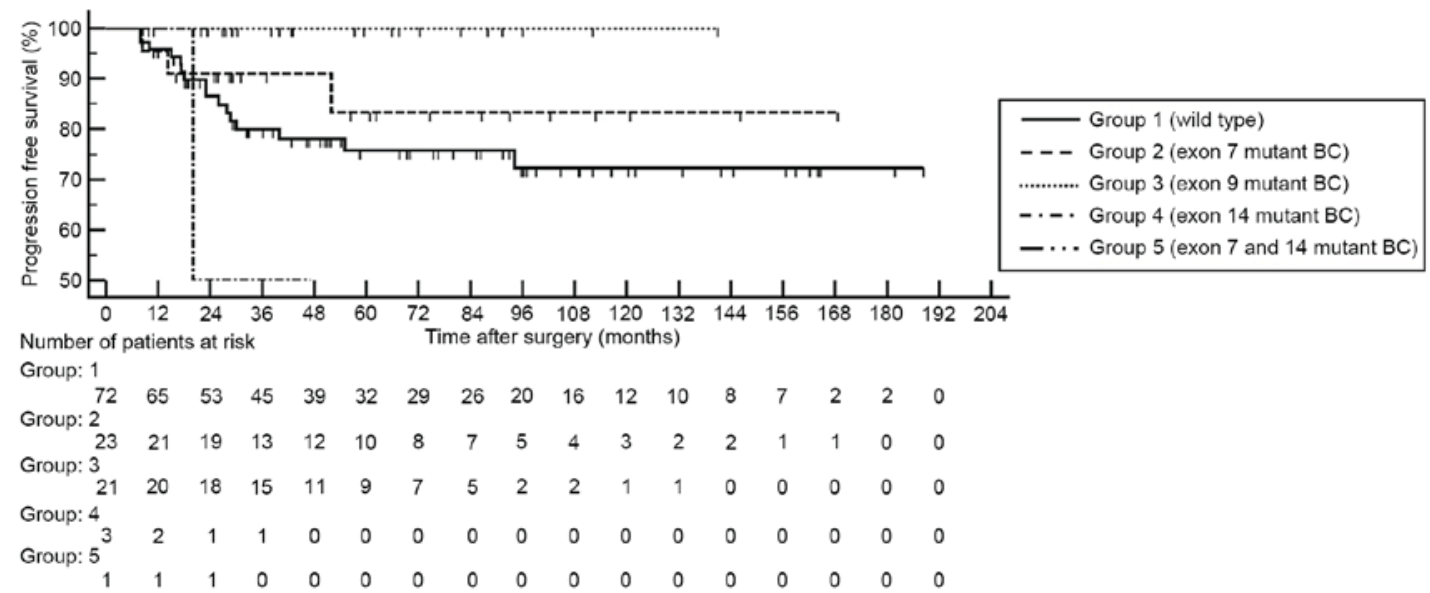

Figure 3. Kaplan-Meier curves for predicting progression-free survival according to $F G F R 3$ mutation status and mutation exon site. FGFR3, fibroblast growth factor receptor 3; $\mathrm{BC}$, bladder cancer.

expression categorized by exon site, which encode various functional domains of FGFR3 protein, including exon 7 (immunoglobulin-like domain: Codon 248, 249), exon 9 (transmembrane domain: Codon 370, 371, 373, and 391) and exon 14 (tyrosine kinase domain: Codon 650). Of note, the present study demonstrated that mutations in exon 7 and 9 revealed significant high FGFR3 mRNA expression levels compare with in the wild type BC. Mutations located in exon 14 did not demonstrate significant difference in FGFR3 mRNA expression level compare with the wild type $\mathrm{BC}$. The present study could not conduct survival analysis due to the limited number of progression events. However, none of the FGFR3 mutations in exon 9 led to disease progression or metastasis. Conversely, among the 3 patients with harboring mutant $\mathrm{BC}$ located in exon 14, 1 patient demonstrated cancer progression within 2 years of short interval. It was suggested that prognostic influences of
FGFR3 mutations may be modulated by the mutation site of the FGFR3 gene, but this requires further investigation.

A possible limitation of the present study is that FGFR3 protein levels were not evaluated. Further studies should include these experiments to better understand the association between activating mutations of FGFR3, mRNA expression level and protein expression level. In addition, the sample size was relatively small, which may reduce the statistical power. Thus, further collaborative studies are required in order to confirm the prognostic role of FGFR3 mutation and gene expression in $\mathrm{pT} 1 \mathrm{BC}$.

In conclusion, the results of the present study suggested that FGFR3 mRNA expression level may be a useful tool for providing a more accurate prognosis for individual patients with pT1 BC. Our preliminary analyses suggested that prognostic influences of FGFR3 mutations may be modulated by 
the mutation site of the FGFR3 gene; however, results are preliminary and thus require validation.

\section{Acknowledgements}

This study was supported by Basic Science Research Program through the National Research Foundation of Korea(NRF) funded by the Ministry of Science, ICT \& Future Planning (grant no. NRF-2015R1A2A2A03004100) and by the International Science and Business Belt Program through the Ministry of Science, ICT and Future Planning (grant no. 2016K000297). The specimens for this study were provided by Chungbuk National University Hospital, a member of the National Biobank of Korea, which is supported by the Ministry of Health, Welfare and Family Affairs. The authors would like to thank Ms. Eun-Ju Shim from the National Biobank of Korea at Chungbuk National University Hospital for sample preparation and technical assistance.

\section{References}

1. Johansson SL and Cohen SM: Epidemiology and etiology of bladder cancer. Semin Surg Oncol 13: 291-298, 1997.

2. Knowles MA and Hurst CD: Molecular biology of bladder cancer: New insights into pathogenesis and clinical diversity. Nat Rev Cancer 15: 25-41, 2015.

3. van Rhijn BW, Burger M, Lotan Y, Solsona E, Stief CG, Sylvester RJ, Witjes JA and Zlotta AR: Recurrence and progression of disease in non-muscle-invasive bladder cancer: From epidemiology to treatment strategy. Eur Urol 56: 430-442, 2009.

4. Kim YH, Kim WT, Jeong P, Ha YS, Kang HW, Yun SJ, Moon SK, Choi YH, Kim IY and Kim WJ: Novel combination markers for predicting survival in patients with muscle invasive bladder cancer: USP18 and DGCR2. J Korean Med Sci 29: 351-356, 2014.

5. Brausi M, Witjes JA, Lamm D, Persad R, Palou J, Colombel M, Buckley R, Soloway M, Akaza $\mathrm{H}$ and Böhle A: A review of current guidelines and best practice recommendations for the management of nonmuscle invasive bladder cancer by the International Bladder Cancer Group. J Urol 186: 2158-2167, 2011.

6. Lotan Y, Shariat SF, Schmitz-Dräger BJ, Sanchez-Carbayo M, Jankevicius F, Racioppi M, Minner SJ, Stöhr B, Bassi PF and Grossman HB: Considerations on implementing diagnostic markers into clinical decision making in bladder cancer. Urol Oncol 28: 441-448, 2010.

7. Mitra AP and Cote RJ: Molecular pathogenesis and diagnostics of bladder cancer. Annu Rev Pathol 4: 251-285, 2009.

8. van Rhijn BW, Zuiverloon TC, Vis AN, Radvanyi F, van Leenders GJ, Ooms BC, Kirkels WJ, Lockwood GA, Boevé ER, Jöbsis AC, et al: Molecular grade (FGFR3/MIB-1) and EORTC risk scores are predictive in primary non-muscle-invasive bladder cancer. Eur Urol 58: 433-441, 2010.

9. Dalbagni G, Presti J, Fair W, Reuter VA and Cordon-Cardo C: Genetic alterations in bladder cancer. Lancet 342: 469-471, 1993.

10. Cheng L, Zhang S, MacLennan GT, Williamson SR, Lopez-Beltran A and Montironi R: Bladder cancer: Translating molecular genetic insights into clinical practice. Hum Pathol 42: 455-481, 2011.

11. Iyer G and Milowsky MI: Fibroblast growth factor receptor-3 in urothelial tumorigenesis. Urol Oncol 31: 303-311, 2013.

12. Jebar AH, Hurst CD, Tomlinson DC, Johnston C, Taylor CF and Knowles MA: FGFR3 and Ras gene mutations are mutually exclusive genetic events in urothelial cell carcinoma. Oncogene 24: 5218-5225, 2005.

13. Pandith AA, Shah ZA and Siddiqi MA: Oncogenic role of fibroblast growth factor receptor 3 in tumorigenesis of urinary bladder cancer. Urol Oncol 31: 398-406, 2013.

14. Cappellen D, De Oliveira C, Ricol D, de Medina S, Bourdin J, Sastre-Garau X, Chopin D, Thiery JP and Radvanyi F: Frequent activating mutations of FGFR3 in human bladder and cervix carcinomas. Nat Genet 23: 18-20, 1999.
15. Shiang R, Thompson LM, Zhu YZ, Church DM, Fielder TJ, Bocian M, Winokur ST and Wasmuth JJ: Mutations in the transmembrane domain of FGFR3 cause the most common genetic form of dwarfism, achondroplasia. Cell 78: 335-342, 1994.

16. Kompier LC, Lurkin I, van der Aa MN, van Rhijn BW, van der Kwast TH and Zwarthoff EC: FGFR3, HRAS, KRAS, NRAS and PIK3CA mutations in bladder cancer and their potential as biomarkers for surveillance and therapy. PLoS One 5: e13821, 2010.

17. Juanpere N, Agell L, Lorenzo M, de Muga S, López-Vilaró L, Murillo R, Mojal S, Serrano S, Lorente JA, Lloreta J and Hernández S: Mutations in FGFR3 and PIK3CA, singly or combined with RAS and AKT1, are associated with AKT but not with MAPK pathway activation in urothelial bladder cancer. Hum Pathol 43: 1573-1582, 2012.

18. Hernández S, López-Knowles E, Lloreta J, Kogevinas M, Jaramillo R, Amorós A, Tardón A, García-Closas R, Serra C, Carrato A, et al: FGFR3 and Tp53 mutations in T1G3 transitional bladder carcinomas: Independent distribution and lack of association with prognosis. Clin Cancer Res 11: 5444-5450, 2005

19. van Rhijn BW, Lurkin I, Radvanyi F, Kirkels WJ, van der Kwast TH and Zwarthoff EC: The fibroblast growth factor receptor 3 (FGFR3) mutation is a strong indicator of superficial bladder cancer with low recurrence rate. Cancer Res 61: 1265-1268, 2001.

20. Neuzillet Y, van Rhijn BW, Prigoda NL, Bapat B, Liu L, Bostrom PJ, Fleshner NE, Gallie BL, Zlotta AR, Jewett MA and van der Kwast TH: FGFR3 mutations, but not FGFR3 expression and FGFR 3 copy-number variations, are associated with favourable non-muscle invasive bladder cancer. Virchows Arch 465: 207-213, 2014.

21. Tomlinson DC, Baldo O, Harnden P and Knowles MA: FGFR3 protein expression and its relationship to mutation status and prognostic variables in bladder cancer. J Pathol 213: 91-98, 2007.

22. Hernández S, López-Knowles E, Lloreta J, Kogevinas M, Amorós A, Tardón A, Carrato A, Serra C, Malats N and Real FX: Prospective study of FGFR3 mutations as a prognostic factor in nonmuscle invasive urothelial bladder carcinomas. J Clin Oncol 24: 3664-3671, 2006

23. van Rhijn BW, van der Kwast TH, Liu L, Fleshner NE, Bostrom PJ, Vis AN, Alkhateeb SS, Bangma CH, Jewett MA, Zwarthoff EC, et al: The FGFR3 mutation is related to favorable pT1 bladder cancer. J Urol 187: 310-314, 2012.

24. Torloni H: Histologic typing of urinary bladder tumors, international histological classification of tumors. World Health Organization, Geneva, 1973.

25. van Oers JM, Lurkin I, van Exsel AJ, Nijsen Y, van Rhijn BW, van der Aa MN, Zwarthoff EC: A simple and fast method for the simultaneous detection of nine fibroblast growth factor receptor 3 mutations in bladder cancer and voided urine. Clin Cancer Res 11: 7743-7748, 2005.

26. Livak KJ and Schmittgen TD: Analysis of relative gene expression data using real-time quantitative PCR and the $2-\Delta \Delta \mathrm{CT}$ method. methods 25: 402-408, 2001.

27. Bernard-Pierrot I, Brams A, Dunois-Lardé C, Caillault A, Diez de Medina SG, Cappellen D, Graff G, Thiery JP, Chopin D, Ricol D and Radvanyi F: Oncogenic properties of the mutated forms of fibroblast growth factor receptor $3 \mathrm{~b}$. Carcinogenesis 27 : 740-747, 2006

28. Guancial EA, Werner L, Bellmunt J, Bamias A, Choueiri TK, Ross R, Schutz FA, Park RS, O'Brien RJ, Hirsch MS, et al: FGFR3 expression in primary and metastatic urothelial carcinoma of the bladder. Cancer Med 3: 835-844, 2014.

29. Billerey C, Chopin D, Aubriot-Lorton MH, Ricol D, Gil Diez de Medina S, Van Rhijn B, Bralet MP, Lefrere-Belda MA, Lahaye JB, Abbou CC, et al: Frequent FGFR3 mutations in papillary non-invasive bladder (pTa) tumors. Am J Pathol 158: 1955-1959, 2001.

30. Burger M, van der Aa MN, van Oers JM, Brinkmann A, van der Kwast TH, Steyerberg EC, Stoehr R, Kirkels WJ, Denzinger S, Wild PJ, et al: Prediction of progression of non-muscle-invasive bladder cancer by WHO 1973 and 2004 grading and by FGFR3 mutation status: A prospective study. Eur Urol 54: 835-844, 2008. 